\title{
The Roles of Siglec7 and Siglec9 on Natural Killer Cells in Virus Infection and Tumour Progression
}

\author{
Yayun Zheng $\mathbb{D}^{1,2,3}$ Xue Ma, ${ }^{4}$ Dongmei Su, ${ }^{1,2}$ Yue Zhang, ${ }^{1,2}$ Lin $Y u\left(\mathbb{D},{ }^{1,2}\right.$ Fangfei Jiang $\mathbb{D},{ }^{1,2}$ \\ Xue Zhou, ${ }^{3}$ Ying Feng $\left(\mathbb{D},{ }^{3}\right.$ and Fang $M a{ }^{10}{ }^{1,2}$ \\ ${ }^{1}$ Center for Translational Medicine, Key Laboratory of Birth Defects and Related Diseases of Women and Children \\ (Sichuan University), Ministry of Education, West China Second University Hospital, Sichuan University, Chengdu, \\ Sichuan 610041, China \\ ${ }^{2}$ Department of Obstetrics and Gynecology, West China Second University Hospital, Sichuan University, Chengdu, \\ Sichuan 610041, China \\ ${ }^{3}$ Department of Histology, Embryology and Neurobiology, West China School of Basic Medical Sciences \& Forensic Medicine, \\ Sichuan University, Chengdu, Sichuan 610041, China \\ ${ }^{4}$ Department of Pediatric Urology, West China University Hospital, Sichuan University, Chengdu, Sichuan 610041, China
}

Correspondence should be addressed to Ying Feng; yingfeng27@scu.edu.cn and Fang Ma; mafangmed@126.com

Received 11 October 2019; Revised 6 March 2020; Accepted 16 March 2020; Published 6 April 2020

Academic Editor: Lihua Duan

Copyright (c) 2020 Yayun Zheng et al. This is an open access article distributed under the Creative Commons Attribution License, which permits unrestricted use, distribution, and reproduction in any medium, provided the original work is properly cited.

\begin{abstract}
The function of natural killer (NK) cells, defending against virus infection and tumour progression, is regulated by multiple activating and inhibiting receptors expressed on NK cells, among which sialic acid-bind immunoglobulin-like lectins (Siglecs) act as a vital inhibitory group. Previous studies have shown that Siglec7 and Siglec9 are expressed on NK cells, which negatively regulate the function of NK cells and modulate the immune response through the interaction of sialic acid-containing ligands. Siglec7 and Siglec9 are very similar in distribution, gene encoding, protein sequences, ligand affinity, and functions in regulating the immune system against virus and cancers, but differences still exist between them. In this review, we aim to discuss the similarities and differences between Siglec7 and Siglec9 and analyze their functions in virus infection and tumour progression in order to develop better anti-viral and anti-tumor immunotherapy in the future.
\end{abstract}

\section{Introduction}

Natural killer (NK) cells are essential innate immune cells which are able to directly kill unhealthy host cells, including virus-infected cells [1] and tumour cells [2]. Due to the downregulation of MHC I of virus-infected cells and tumour cells, the target cells can escape from the specific recognition of T cells [3]. However, NK cell-mediated cytotoxicity does not require antigenic stimulation and is not restricted by MHC I molecules. Various activating receptors, such as NKp30, NKp44, NKp46, CD16, NKG2C, and NKG2E, are involved in the NK-mediated cytotoxicity. Not only activating receptor interaction with ligands can induce NK cells to kill target cells but also inhibitory receptors take part in regulating the cellular cytotoxicity exerted by NK cells.
Siglecs are important fragments of receptors, which regulate inhibitory signal conduction on NK cells [4]. They are characterized subset of immunoglobulin superfamily and one family of type I transmembrane proteins of I-lectin. Sialic acid (Sia), on the one hand, is a group of 9-carbonbacknone monosaccharide and is necessary for immunomodulation; on the other hand, it is hydrophilic and negatively charged and widely expressed at the terminal of the protein or lipid on the cell surface and secretory proteins $[5,6]$. Siglecs are critical receptors of Sia. Siglecs promote cell-cell recognition and modulate the cytotoxicity of NK cell towards virus and tumour by binding to the Sia residue of the glycoconjugates on target cells. According to the evolution, Siglecs can be divided into two groups. The conserved Siglecs include sialoadhesin (Siglec1), CD22 (Siglec2), MAG 
TABLE 1: The expression and ligand affinity of Siglec7 and Siglec9 in human immune cells.

\begin{tabular}{lccccccccccc}
\hline & & \multicolumn{4}{c}{} & \multicolumn{4}{c}{ Expression } & & \\
& Neutrophil & NK cells & T cells & B cells & Monocyte & Macrophage & Eosinophil & Basophils & $\begin{array}{c}\text { Dendritic } \\
\text { cells }\end{array}$ & Ligand affinity \\
\hline Siglec7 & + & + & + & - & + & + & + & + & + & $\alpha 2,8>\alpha 2,6>\alpha 2,3$ \\
Siglec9 & + & + & + & + & + & + & - & - & + & $\alpha 2,6 \alpha 2,3$ sulfated residues \\
\hline
\end{tabular}

(Siglec4), and Siglec15. Another group involves a group of rapidly evolving Siglecs, named CD33-related Siglecs. In human, CD33-related Siglecs consist of CD33 (Siglec3), Siglec5, Siglec6, Siglec7, Siglec8, Siglec9, Siglec10, Siglec11, Siglec14, and Siglec16. However, due to no homology between human and mouse, CD33-related Siglecs in mouse comprise CD33 (Siglec3), Siglec-E, Siglec-F, Siglec-G, and Siglec-H.

Siglecs play important roles in mediating immune response. For example, CD22 (Siglec-2), specifically expressed on B cells, interacts with its ligand, $\alpha 2,6$-linked Sia on adjacent cells, inhibiting cell activating signal transduction, calcium mobilization and $\mathrm{B}$ cell receptor activation [7]. Besides, antibody blocking of Siglec8 can induce caspase-3like apoptosis and inhibit eosinophil viability [8]. Moreover, the interaction between Siglec7 and Sia,or between Siglec9 and Sia leads to the phosphorylation of immunoreceptor tyrosine-based inhibition motif (ITIM), which can suppress the cytotoxicity of NK cells. Whereas the most prominent Siglecs of immune regulation on NK cells are Siglec7 and Siglec9 [9], this review will focus on the current research progress on the similarities and differences between Siglec7 and Siglec9 and their functions in tumour and virus infection progression.

\section{The Distribution of Siglec7 and Siglec9}

Siglec7 and Siglec9 are mainly expressed in immune cells. Siglec7 is primitively reported to be expressed on NK cells, especially in CD56 ${ }^{\text {bright }}$ cells. In addition, a minor subset of $\mathrm{CD}^{+} \mathrm{T}$ cells, representing cytotoxic $\mathrm{T}$ cells, was also confirmed to express Siglec7. The expression level in granulocyte was reported to be lower than monocyte, which was lower than the lymphocyte's [10]. In the past years, it was also proved that Siglec7 was expressed on macrophages [11], dendritic cells [12], mast cells, and basophils [13] (Table 1). Siglec9 is expressed quite broadly among human blood leukocytes, including monocytes, neutrophils, B cells, NK cells, and minor subsets of $\mathrm{T}$ cells [14]. In peripheral blood, the expression of Siglec9 is predominantly on neutrophils [1517] and followed by NK cells, B cells, and monocytes [11]. Siglec9 was also detected on tissue resident macrophages at low level $[11,18,19]$ (Table 1). And it was reported that the Siglec9 level on neutrophil rose higher than adults during the neonatal period, but the high level withdrew in one month after birth. This may be caused by the infection of the bacterium, Group B Streptococcus (GBS), presenting mostly in neonatal period [20]. Although the cDNA of Siglec9 was cloned from promyeloblast, HL60, little evidence showed that HL60 cells express the protein of Siglec9. It indi- cates that Siglec9 is possibly expressed in advanced differentiating immune cells [18].

Apart from the immune system, Siglec7 is also found in other cells, while the expression of Siglec9 in other cells has not been reported. Nguyen et al. illustrated that Siglec7 was detected on platelets of healthy donators, and the crosslinking Siglec7 with its ligand, ganglioside, resulted in platelet apoptosis [21]. Therefore, Siglec7 may be a potential therapy target of platelet diseases. Moreover, Dharmadhikari et al. revealed that Siglec 7 detected in $\beta$-cells of human pancreatic islets may participate in glycan metabolism by inhibiting $\beta$ cell apoptosis [22].

\section{The Gene and Protein Sequence Comparison of Siglec7 and Siglec9}

With around $84 \%$ identity in coding sequence, Siglec7 (p75/AIRM1 or CD328) and Siglec9 encoding genes are both located in chromosome 19q13.3-13.4 (Figure 1(a)). Fulllength cDNA sequence of Siglec7 was firstly cloned from a human primary dendritic cell cDNA library by Nicoll and his colleagues in 1999, and it is 1748 base pair long encoding a 467-amino acid protein [10]. While Siglec9's full-length cDNA sequence was found in the cDNA library of human acute promyelocytic leukemia cells one year after Siglec7, which contain a 1392-nucleotide open reading frame and produces a 463-amino-acid protein [23].

The proteins of Siglec7 and Siglec9 are both type I membrane proteins of the Ig superfamily. Siglec7 is a $75 \mathrm{kDa}$ protein (the monomer of Siglec7 is reported to be about $65 \mathrm{kDa}$ ) [10], while the protein of Siglec9 is $50.1 \mathrm{kDa}$ [23]. Although there is a difference of molecular weight between Siglec7 and Siglec9, the protein structure of Siglec7 is very similar to that of Siglec9 (Figure 1(b), Table 2). They both consist of an extracellular region, a transmembrane region, and a cytoplasmic tail. The extracellular region of both Siglec7 and Siglec9 contains a hydrophobic signal peptide and three Ig-like domains including one $\mathrm{N}$ terminal V-set domain and two C2-set domains. The hydrophobic signal peptide in the extracellular region has 18 amino acids in Siglec7 and 17 amino acids in Siglec9, respectively. In the three Ig-like domains of both Siglec7 and Siglec9, there are eight potential $\mathrm{N}$-linked glycosylation sites [10, 14]. Whereas when Siglec7 was treated with $N$-glycosidase, it showed a protein backbone of $48 \mathrm{kD}$ [24].Except for the $\mathrm{N}$ linked glycosylation sites, the Sia binding sites exist in both of the proteins. The binding of Siglecs with Sia requires multiple sites to function simultaneously (please see Section 4 below for details). 


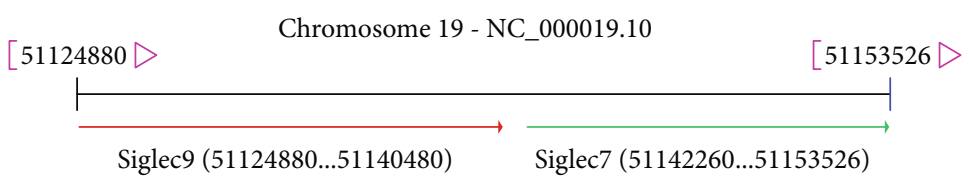

(a)

CLUSTAL W (1.83) multiple sequence alignment

\begin{abstract}
sp $\mid$ Q9Y286 $\mid$ SIGL7_HLMAN MLLLLLLPLLWGRERVEGQKSNRKDYSLTMQSSVTVQEGMCVHVRCSFSYPVDSQTDSDP-VHGYWFRAGNDISWKAPVA
sp $\mid$ Q9Y336|SIGL9_HUMAN MLL-LLLPLLWGRERAEGQTSK----LLTMQSSVTVQEGLCVHVPCSFSYPSHGWIYPGPVVHGYWFREGANTDQDAPVA

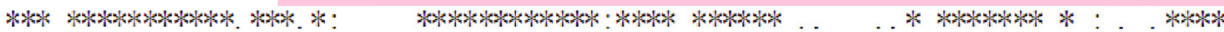

\begin{tabular}{l|l|l|l|}
$\mathrm{sp}$ & Q9Y286 & SIGL7_HUMAN \\
$\mathrm{sp}$ & Q9Y336 & SIGL9_HUMAN
\end{tabular} TNXPAWAVQEETRDRFHLLGDPQTWNCTLSIRDARMSDACRYFFRMEKGNIKWNYKYDQLSWWTALTHRPNILIPGILE TNXPARAVWEETRDRFHLLGDPHT KNCTLSIRDARRSDACRYFFRMEKGSI KWNYKHHRLSWWTALTHRPNIL IPGILE $* * * * * * * * * * * * * * * * * * * * * ; * * * * * * * * * * * * * * * * * * * * * * * * * * *, * * * * * * ;: ; * * * * * * * * * * * * * * * * * * * * * * *$

sp Q9Y286 $\mid$ SIGL7_HLMAN sp $\mid$ Q9Y336 $\mid$ SIGL9_HLMAN

SGCFQTLTCSVPWACEQGTPPMISWMGTSVSPLHPSTTRSSVLTLIPQPQHHGTSLTCQVTLPGAGVTTRRTIQLVVIYP SGCPQLLTCSVPWACEQGTPPMISWIGTSVSPLDPSTTRSSVLTLIPQPQDHGTSLTCQVTFPGASVTTAKTVHLVVSYP

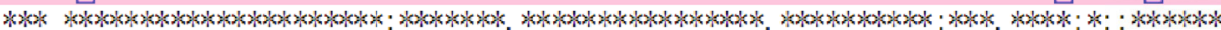

sp $\mid$ Q9Y286 $\mid$ SIGL7_HUMAN sp Q9Y336 |SIGL9_HUMAN

PQNLTVTVFQGEGTASTALGNSSSLSVLEGQSLRLVCAV---DSNPPARLSWTWRSLTLYPSQPSNPLVLELQ-VHLGDE PQNLTMTVFQGDGTVSTVLGNGSSLSLPEGQSLRLVCAVDAVDSNPPARLSLSWRGLTLCPSQPSNPGVLELPWVHLRDA

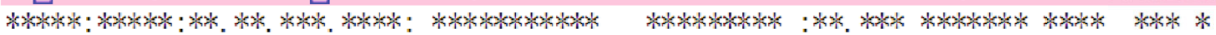

sp Q9Y286 SIGL7_HUMAN sp Q9Y336 $\mid$ SIGL9_HLMAN

\title{
GEFTCRAQNSLGSQHVSLRLSLQQEYTGKMRPVSGVLLGAVGGAGATALVFLSFCV IFIVVRSCRKKSARPAADVGDIGM AEFTCRAQNPLGSQQVYLVSLQSKAT------SGVTQGVVGGAGATALVFLSFCV IFVVVRSCRKKSARPAAGVGDTGI

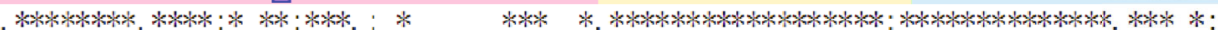

sp $\mid$ Q9Y286 $\mid$ SIGL7_HLMAN

KDANIIRGSASQGNLTESWADDNPRHHG--LAAHSSGEEREIQYAPLSFHKGEPQDLSGQEATYNEYSE IKIPK EDANAVRGSASQGPLTEPWAEDSPPDQPPPASARSSVGEGELQYASLSFQMVKPWDSRGQEATIIEYSE IKIHR

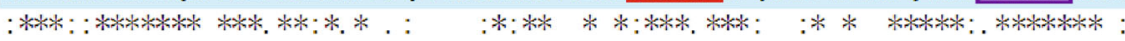

Extracellular region

Transmembrane region

Cytoplasmic region

(b)

Figure 1: (a) The gene location of Siglec7 and Siglec9. They are both mapped in the chromosome 19q13.3-13.4. (b) The sequence alignment of protein Siglec7 and Siglec9. The protein sequences are from uniport (The UniProtKB of Siglec7 Human: Q9Y286; Siglec9 Human: Q9Y336). The topological domains are colored in pink, light yellow, and blue, representing the extracellular region, transmembrane region, and cytoplasmic region, respectively. Asterisk: indicating a conserved amino acid in all sequences; colon: indicating the position of a sequence alignment composed of residues having similar physicochemical properties; point: indicating the column of the multiple sequence alignment in which the semiconservative substitution was observed. The dark blue frames indicate the eight glycosylation sites. The green frame represents the Sia binding site, and the yellow frame indicates the Sia binding region. The red frame denotes the ITIM motif, and the purple frame signifies the ITIM-like motif.

TABLe 2: Amino acid sequence analysis of Siglec7 and Siglec9 in human immune cells.

\begin{tabular}{lcccccc}
\hline & \multirow{2}{*}{ Signal peptide } & \multicolumn{3}{c}{ Extracellular region } & \multirow{2}{*}{ Transmembrane region } & \multicolumn{2}{c}{ Cytoplasmic region } \\
& & Ig-like V-type & Ig-like C2-type 1 & Ig-like C2-type 2 & ITIM motif & ITIM-like motif \\
\hline Siglec7 & $1-18$ & $39-122$ & $150-233$ & $240-336$ & $354-376$ & $435-440$ \\
Siglec9 & $1-17$ & $20-140$ & $146-229$ & $236-336$ & $349-369$ & $458-463$ \\
\hline
\end{tabular}

Although Siglec7 and Siglec9 are reported as type I transmembrane proteins, Varchetta et al.'s group discovered a soluble Siglec7 (sSiglec7) without a transmembrane region in circular peripheral blood [25]. Zeng et al.'s group also unveiled a soluble Siglec9 (sSiglec9) in the plasma, which can induce the oxidative stress, and the expression can be increased by TNF- $\alpha$, IL-6, and IL- 8 [26]. Matsumoto et al.'s group also detected the injected-sSiglec 9 in the inflamed tissue of the mouse paws and all digits after intravenously administering recombinant human Siglec9 into arthritis mice. They also proved that sSiglec 9 can suppress arthritis in the mouse model [27]. Siglec-E is the mouse ortholog of human Siglec-9. However, whether Siglec-E participated in suppressing arthritis was not mentioned in the study. While human sSiglec9 transgenic mice presented resistance against GBS infection [28], as well as anti-tumour 
benefit against mammary tumour cells [29], the structure of sSiglec9 has yet not been well characterized.Hence, further study is required.

The cytoplasmic regions of Siglec7 and Siglec9 both have two important functional domains: a membrane proximal ITIM domain and a membrane-distal motif. Pro ${ }^{439}$ in the proximal motif and $\mathrm{Asn}^{458}$ in the distal motif of Siglec7 both contribute to recruiting phosphatases [30]. The ITIM motif in Siglec9 is very similar to Siglec7, Ile ${ }^{435}$-Gln-Tyr-AlaPro-Leu $^{440}$ in Siglec7 and Leu ${ }^{431}-$ Gln-Tyr-Ala-Ser-Leu ${ }^{436}$ in Siglec9. And the membrane-distal motif of Siglec9 $\mathrm{Thr}^{454}$-Glu-Tyr-Ser-Glu-Ile ${ }^{459}$ is an ITIM-like motif, which is $\mathrm{Asn}^{458}$-Glu-Tyr-Ser-Glu-Ile ${ }^{463}$ in Siglec7 [10, 18].

\section{The Ligand Affinity of Siglec7 and Siglec9}

Although comparison of the amino acid sequences between Siglec7 and Siglec9 results in a similarity up to $98 \%$ (comparison tool: T-coffee http://tcoffee.vital-it.ch/), ligand affinity differences still exist between Siglec7 and Siglec9. It was reported that Siglec7 binds to $\alpha 2,8$-sialyl residue with the highest affinity, while binds to $\alpha 2,6$-sialyl residue with lower affinity and $\alpha 2,3$-sialyl residue with the lowest affinity [31, 32]. Meanwhile, Siglec 9 prefers to bind to $\alpha 2,6$-sialyl and $\alpha 2,3$-sialyl residues [14, 31].

Siglec7 and Siglec9 can also bind to sulfated sialyl Lewis ${ }^{\mathrm{x}}$, which was proved on normal colonic epithelial cells. And the expression of sulfated sialyl Lewis ${ }^{\mathrm{x}}$ decreased when these cells become colonic cancer cells. The ligand affinity was confirmed by a consortium of functional glycomics using ELISA on molecular level (http://www.functionalglycomics.org) and flow cytometric analyses on cell level in Miyazaki et al.'s group [11]. The ELISA results showed that Siglec-7 was the seventh reactive to sialyl 6-sulfo Lewis ${ }^{\mathrm{x}}$, while Siglec9 was the most reactive. However, cell level results showed that sialyl 6-sulfo Lewis ${ }^{\mathrm{x}}$ was detected strongly binding to Siglec7 and hardly binding to Siglec9. The contradictory findings may be due to that ELISA results on a molecular level may not always be consistent with the cell experiments, and this requires further study.

The Sia binding characteristics of Siglec7 and Siglec9 are determined by specific amino acids. Yamaji et al.'s group exchanged the V-set domain of Asn (70)-Lys (75) between Siglec7 and Siglec9, resulting in loss of the original binding specificities and gaining of each other's binding properties. Proved by aforementioned evidence, they further highlighted that the affinity specificity of Siglec 7 and Siglec9 was determined by the C-C loop of the glycan binding domain [33], whereas the Arg124 in Siglec7 and Arg120 in Siglec9 was the definite amino acid for binding to Sia. It was reported that Siglec-9 transiently transfecting to COS cells was able to recognize red blood cells, but COS cells expressing Arg120mutant Siglec9 could not bind to red blood cells. This result indicated that the stable salt bridge connecting Arg and Sia is necessary for binding $[18,31]$.

Moreover, Malaker et al. proved that cells treated with secreted protease of $\mathrm{C} 1$ esterase inhibitor (StcE) had a decreased affinity with Siglec7, but the binding to Siglec9 was not affected. StcE is a bacterial protease from E. coli and selective for mucin-domain cleavage by identifying discrete peptide- and glycan-based motifs. The main feature of the mucin domain is the high frequency of Ser and Thr residues that are $O$-glycosylated by $\alpha-N$-acetylgalactosamine $(\alpha-$ GalNAc) [34]. This phenomime indicates that the binding between Siglec7 and cells depends on the mucin domain, and the binding between Siglec9 and cells may rely on some other motifs rather than mucin domain. Besides, StcE was reported to mainly cut densely $O$-glycosylated proteins and has no reactivity with $N$-glycosylated proteins and sparsely $\mathrm{O}$-glycosylated [35]. The difference in ligand affinity between Siglec7 and Siglec9 may not only be due to the differences in sialylation site but also be due to the differences in $\mathrm{N}$-glycosylation or $\mathrm{O}$-glycosylation, even the density of glycosylation. Taken together, accumulating evidences indicate Siglec7 and Siglec9 have diverse ligand affinity which, to some extent, explains the different function of the two lectins in immune cells.

\section{The Function of Siglec7 and Siglec9 in NK Cells}

Siglec7, Siglec9, and Siglec-17 are expressed in NK cells and play important roles in inhibitory signal transducing through Sia-dependent binding. Siglec7, first detected in NK cells in 1999, serves as an inhibitory receptor mediating Sia-dependent ligand recognition $[10,24]$. Shao et al. proved that the Siglec $7^{+}$subgroup of NK cells produces more CD107a degranulation and secretes more cytokines, such as IFN- $\gamma$ and TNF- $\alpha$, and they considered Siglec7 as a marker of a higher function group of NK cells [36]. Siglec9, which is highly related to Siglec7, was first found in NK cells in $2000[14,18]$. It has been proved that the expression of Siglec9 happened in the early stage of NK cell differentiation from $\mathrm{CD} 56^{\text {bright }}$ to $\mathrm{CD} 56^{\mathrm{dim}}$. Jandus et al. indicated that Siglec9 might be a maker for an early maturity group of NK cells, which are less cytotoxic but more chemotactic [9]. It was previously reported that Siglec7 was expressed on all NK cells $[10,24]$. However, more and more evidences show that there are Siglec $7^{-}$NK cell subsets [10, 36, 37]. Shao et al. reported that Siglec7 was preferentially expressed on mature NK cells, and Siglec $7^{+}$NK cells express more activating receptors and less inhibitory receptors. They believed that Siglec7 may be defined a more cytotoxic group of NK cells [36]. And Siglec9 is expressed on $30-40 \%$ of CD56 ${ }^{\mathrm{dim}} \mathrm{NK}$ cells [19] and shows very weakly positive on CD56 ${ }^{\text {bright }} \mathrm{NK}$ cells [18]. Except for immune function, it is showed that the expression of Siglec7 decreased on NK cells among the obese population $\left(\mathrm{BMI}>30 \mathrm{~kg} / \mathrm{m}^{2}\right)$, which was not applicable to Siglec9 [38]. However, whether Siglec7 participates in lipid metabolism remains unclear.

Abundant evidences have proved that Siglec7 and Siglec9 exhibit inhibitory roles in regulating the immune balance $[39,40]$. The important functional domain of both Siglecs 7 and Siglec9 is the membrane-proximal ITIM, mediating the inhibitory signaling. Siglecs crosstalk with Sia of the glycoconjugates through its N-terminal Sia-binding domain and mediate cell-cell recognition, which induce tyrosine phosphorylation. ITIMs are able to provide docking sites 


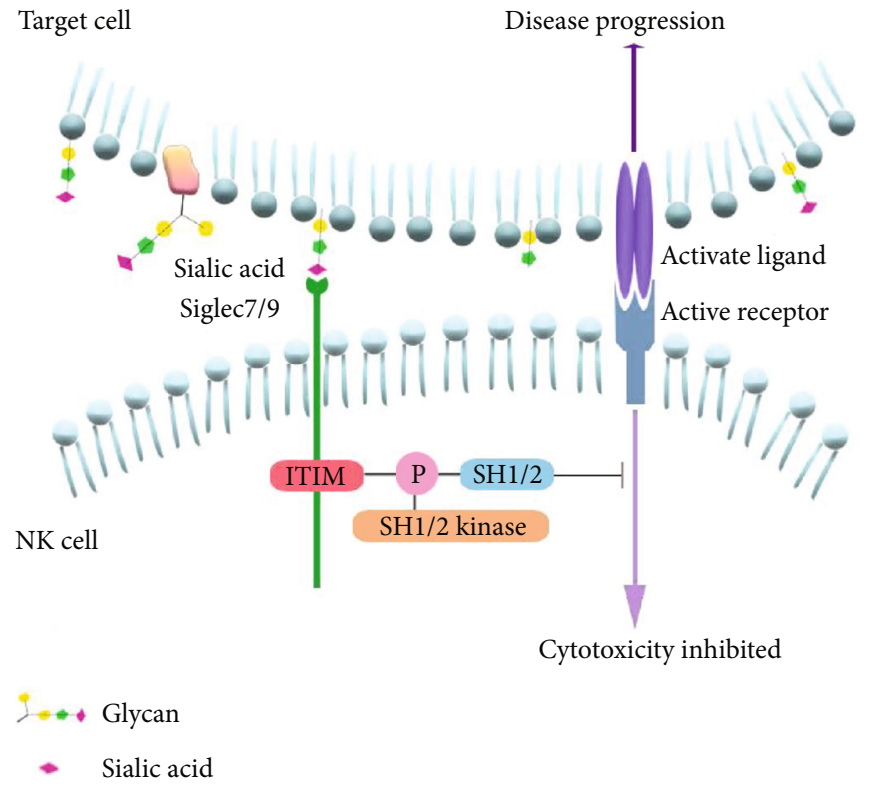

FIgURE 2: Siglec-7/9, cross-talking with sialic acids (Sia) on the surface of target cells, can inhibit the cytotoxicity of NK cells. The interaction between siglec7/9 and Sia can lead to the phosphorylation of ITIM and recruit SHP-1 and SHP-2, which will suppress the NK cell activation, and resulting in disease progression.

for Src homology 1/2domain containing cytoplasmic phosphatases(SHP-1/2) when tyrosine phosphorylated, which is critical for signal delivery $[41,42]$. Through the interaction with Sia, Siglec7 can reduce the cytotoxicity of NK cells towards the target cells through the ITIM recruiting SHP-1/2, blocking signal conduction pathway, and playing negative regulation (Figure 2) [43]. Here, we aim to discuss the functions of Siglec7 and Siglec9 in virus infection and tumour progression.

5.1. Siglec7 Involves in Human Immunodeficiency Virus-1 (HIV-1), Hepatitis B Virus (HBV), and Hepatitis C Virus (HCV) Infection, While Siglec 9 Participates in the HBV Replication. Siglec7 is involved in HIV-1 infection, which usually accompanies the changes of Siglec7 expressions on NK cells while maintaining the total number of NK cells in the peripheral blood $[44,45]$. Brunetta et al.'s group indicated that Siglec7 may be a biomarker of disorder functional subsets of NK cells and HIV-1 infection, because they found that reduced number of Siglec $7^{+}$NK cell subgroup was related to the high level of HIV-1 replication [44]. They also believed that the downregulation of Siglec7 on NK cells was due to the decreased size of the Siglec $7^{+}$NK cell subgroup and increased amount of the Siglec7 $7^{-}$NK cells, while the total number of NK cells in the peripheral blood was not reduced. Moreover, based on the study of Siglecs endocytic function [46], Brunetta et al. provided another hypothesis that Siglec7 may bind to HIV-1 envelope (Env) glycoprotein 120 (gp120), triggering the endocytic process of siglec7 [45]. Zulu et al.'s group discovered a decrease of Siglec7 on NK cells in most of HIV-1 samples from the peripheral blood collected from healthy donors, chronic viremic, long-term nonprogressor (LTNP), and early viremic HIV-1-infected patients. They also found out that Siglec7 in CD56 ${ }^{\mathrm{dim}}$ NK cells in viremic patients decreased with comparison to healthy donors [47], whereas Brunetta et al.'s group proved that antiretroviral therapy can rescue the expression of Siglec7 [44]. And in vitro studies showed that recombinant human Siglec7 bound to sialyl residues of HIV-1 envelope (Env) glycoprotein 120 (gp120), which mediated virus entrying into target cells $[25,48]$. It revealed that Sia residues on gp 120 can protect the virus through Siglec7 from the human immune system attacking and promote virus replication and disease progression. However, according to Varchetta et al.'s group, an increase level of serum sSiglec7 was detected in AIDS patients compared to healthy donors [25]. Siglec7 on the NK cell surface was suggested to be masked by virus, and the serum sSiglec7 may be from the apoptosis NK cells [25, 44, 49].

Besides, Siglec7 may contribute to HCV and HBV infection. Similar to the expression profile in HIV-1 patients, the expression of Siglec7 on NK cells declined in HCV-infected and $\mathrm{HBV}$-infected patients, whereas the serum sSiglec7 level increased $[37,50]$. The serum sSiglec7 level was associated with the $\mathrm{HBV} / \mathrm{HCV}$ level and negatively correlated with Siglec $7^{+}$NK cells.

Siglec9 participates in the hepatitis B virus (HBV) replication and is involved in the dysfunction of NK cells. Zhao et al.'s group pointed out that the level of Siglec9 on NK cells in HBV-infected patients decreased. And the expression level of Siglec9 was negatively correlated with virus replication. Moreover, the Siglec $9^{+}$NK cells of $\mathrm{HBV}$-infected patients show a higher level of several activating receptors than the Siglec9 ${ }^{-}$NK cells do. However, blocking Siglec9 on NK cells of HBV-infected patients can increase IFN- $\gamma$, TNF- $\alpha$ secretion, and CD107a degranulation [51]. And the expression profile of Siglec9 has not been reported in the infection process in HIV and HCV so far. It would be interesting to 
analyze the glycol-structure differences between these viruses to reveal the role of Siglec7 and Siglec9 in the above viral infection.

\subsection{Siglec7 and Siglec9 Binding to Sia Helps Cancer Cells} Escape from Immune Surveillance. One of the hotspots in Siglec7/9 researches is their functions in the interaction of cancer cells and NK cells, due to its anti-tumour effects. NK cells participate in killing tumour cells through the specific lysis of target cells and the secretion of cytokine, such as IFN- $\gamma$ and TNF- $\alpha$. Siglec7 and Siglec9 expressed on NK cells and their ligands play important roles in promoting the process of proliferation and migration of tumour cells. The ligands recognized by recognition Siglec7 and Siglec9, Sia, is the glycan chain ending residue of glycolipids and glycoproteins. We introduce the specific roles of Siglecs binding with Sia from the following three aspects: the total Sia: the sialy-glycolipids, ganglioside, and mucins (MUC), a family of heavily sialylated glycoproteins.

Firstly, Sia, the ligand of Siglec7 and Siglec9 expressed on the surfaces of tumour cells, is correlating with the immune evasion in cancer [52]. Jandus et al.'s group reported that Sia was widely expressed on a great number of different organizational tumour cells, which can protect tumour cells from NK cell-mediated cytotoxicity. As Hudak et al.'s group claimed, Sia-equipped glycan can protect tumour cells from the immune system attacking through Sia-Siglec7 interaction [40]. It is worth mentioning that the hypersialylation tumour cells are capable of binding to the Siglec9 on NK cells and modulating immunosurveillance. Jandus et al.'s group also revealed that Siglec $9^{+} \mathrm{NK}$ cells expressed more inhibitory receptors (KIR and ILT2) and exhibited a less cytotoxicity towards tumour cells. Treating tumour cells with neuraminidase enhanced the NK cell lysis through the degranulation and the secretion of cytokines [9]. Pearce and Laubli have researched one of the main forms of Sia expressed in mammals: the $\mathrm{N}$-glycolylneuraminic acid (Neu5Gc) on the surfaces of tumour cells. Neu5Gc cannot be synthesized by humans, because the human body is lacking of CMP-N-acetylneuraminic acid hydroxylase (the enzyme used to transfer CMPNeu5Gc from CMP-N-acetylneuraminic (CMP-Neu5Ac) acid [53]). But Neu5Gc can be absorbed from the food and becomes the materials of glycan for the human [54]. Moreover, Neu5Gc can enhance the inflammatory response induced by anti-Neu5Gc [55-57].

Secondly, Siglec7 can interact with ganglioside which is composed of glycosphingolipid with a group of Sia. Kawasaki et al.'s group reported that Siglec7 on NK cells bound to one of the major gangliosides expressed on the surface of renal cell carcinoma (RCC) cells: the disialosyl globopentaosylceramide (DSGb5), which lead to a reduction of NK cell cytotoxicity towards RCC cells. DSGb5 then further promoted RCC metastasis and migration potential [58]. In addition to DSGb5, Siglec7 also interacts with GD3, a ganglioside expressed on tumour cells. Siglec7-GD3 interaction suppresses the NK cell killing activity. Thus, with its highly expressed GD3 ganglioside, melanoma is deemed to escape from NK cell cytotoxicity. And through the trans- fection of GD3 synthase to the cell lines without the expression of recombinant Siglec7-Fc protein ligands, the cytotoxicity of NK cells to mastocytoma, P815, and colorectal adenocarcinoma cell, DLD-1, was inhibited [43, 59].

Thirdly, Siglec 9 can interact with transmembrane epithelial MUC, the heavily glycosylated proteins mainly produced by epithelial tissues, such as MUC1 and MUC16, which leads to immune evasion modulation. MUC1 is overexpressed on adenocarcinomas and hematological cancers [60-62]. MUC1 can induce the growth of tumour cells through recruiting $\beta$-catenin binding to its $\mathrm{C}$-terminal domain [63]. MUC1-sialylated O-linked glycans on tumour cells binding to Siglec9 did not recruit SHP-1 or SHP-2 but induced calcium flux that lead to the activation of MEKERK kinases [64]. Similar to MUC1, Siglec9 can interact with MUC16 expressed on epithelial ovarian cancer cells, protecting tumour cells from immune attacking. Siglec9 promoted tumour cell adhesion process through the recognition of MUC16 glycans which contain $\alpha 2,3$-linked Sia, the ligand of Siglec9 [19].

The fact that tumour cells escape from NK cell attack through the interaction of cell surface sialyl-decoration with Siglec7 and Siglec9 on NK cells makes the two lectins promising target in antitumor drug development. Recently, highaffinity low molecular weight Siglec7 ligands are artificially designed and synthesized. The synthetic ligands can weaken the interactions between Siglec7 and its tumour ligands and, hence, inhibit cancer immune evasion [40, 65].

\section{Conclusion and Outlook}

Although Siglec7 and Siglec9 show high similarities in the distribution, gene encoding, protein sequences, ligand affinity, and functions, differences still exist between them. The study of Siglec7 expressed on NK cells is clearer, but the study of Siglec9 on NK cells is lacking. Although Siglec7 and Siglec9 are usually believed to play negative roles in mediating lysis and cytokine secretion in NK cells through Siglecs-ligand recognition, an active role of Siglec7 has been reported as the cross-linking of Siglec7 by a specific antibody that can upregulate the inflammatory cytokines and chemokines in monocytes [66], whereas little research has indicated the active function of Siglec9. With this regard, whether Siglec7 and Siglec 9 possess a positive property in immune response and how they play active roles in regulating immune system require further confirmation.

Siglec7 was proved to be related to HIV, HBV, and $\mathrm{HCV}$ infection, but only the function of Siglec9 in HBV was reported. The evidence that Siglec9 expressed on NK cells is involved in the immune response to HIV and HCV infection needs further study. There may be structural differences of glycan ligands among HIV, HBV, and HCV, resulting in insufficient recognition of HIV and HCV by Siglec9. Although there are abundant evidences that show Siglec7 and Siglec9 expressed on NK cells are related to the HIV, HBV, and HCV infection, the exact mechanism of Siglec7 and Siglec9 involve in these viral infections requires further investigation. 
Siglec7 and Siglec9 binding to the ligands helps the target cells to inhibit the NK cells and also evade immune surveillance. In antivirus and antitumour immunotherapy, the inhibitory effect of Siglec7 and Siglec9 can be blocked by either adding antibodies of the two proteins or the elimination of cell surface ligands of the two. In addition, the regulatory molecules of Siglecs and sialylation can be considered. A glycocalyx engineering approach is created to help tumour cells suppress NK cell lysis. However, whether it contributes to defecting pathogen-infected or tumour deterioration in vivo demands further studies.

\section{Conflicts of Interest}

The authors declare that they have no conflict of interest.

\section{Authors' Contributions}

Yayun Zheng and Xue Ma are co-first authors.

\section{Acknowledgments}

This work was supported by the National Key Research and Development Program of China (Grant number 2018YFC1002803) and the National Science Foundation of China (Grant number 31771662).

\section{References}

[1] B. Oliviero, S. Mantovani, S. Varchetta et al., "Hepatitis C virus-induced NK cell activation causes metzincin-mediated CD16 cleavage and impaired antibody-dependent cytotoxicity," Journal of Hepatology, vol. 66, no. 6, pp. 1130-1137, 2017.

[2] I. Prager, C. Liesche, H. van Ooijen et al., "NK cells switch from granzyme B to death receptor-mediated cytotoxicity during serial killing," The Journal of Experimental Medicine, vol. 216, no. 9, pp. 2113-2127, 2019.

[3] L. A. M. Cornelissen, A. Blanas, J. C. van der Horst et al., "Disruption of sialic acid metabolism drives tumor growth by augmenting $\mathrm{CD}^{+} \mathrm{T}$ cell apoptosis," International Journal of Cancer, vol. 144, no. 9, pp. 2290-2302, 2019.

[4] J. Daly, M. Carlsten, and M. O'Dwyer, "Sugar free: novel immunotherapeutic approaches targeting Siglecs and Sialic acids to enhance natural killer cell cytotoxicity against cancer," Frontiers in Immunology, vol. 10, p. 1047, 2019.

[5] R. Schauer, "Achievements and challenges of sialic acid research," Glycoconjugate Journal, vol. 17, no. 7-9, pp. 485499, 2000.

[6] A. Varki, "Glycan-based interactions involving vertebrate sialic-acid-recognizing proteins," Nature, vol. 446, no. 7139, pp. 1023-1029, 2007.

[7] K. L. Otipoby, K. E. Draves, and E. A. Clark, "CD22 regulates B cell receptor-mediated signals via two domains that independently recruit Grb2 and SHP-1," The Journal of Biological Chemistry, vol. 276, no. 47, pp. 44315-44322, 2001.

[8] E. Nutku, H. Aizawa, S. A. Hudson, and B. S. Bochner, "Ligation of Siglec-8: a selective mechanism for induction of human eosinophil apoptosis," Blood, vol. 101, no. 12, pp. 5014-5020, 2003.

[9] C. Jandus, K. F. Boligan, O. Chijioke et al., "Interactions between Siglec-7/9 receptors and ligands influence NK cell- dependent tumor immunosurveillance," The Journal of Clinical Investigation, vol. 124, no. 4, pp. 1810-1820, 2014.

[10] G. Nicoll, J. Ni, D. Liu et al., "Identification and characterization of a novel siglec, siglec-7, expressed by human natural killer cells and monocytes," The Journal of Biological Chemistry, vol. 274, no. 48, pp. 34089-34095, 1999.

[11] K. Miyazaki, K. Sakuma, Y. I. Kawamura et al., "Colonic epithelial cells express specific ligands for mucosal macrophage immunosuppressive receptors siglec-7 and -9," Journal of Immunology, vol. 188, no. 9, pp. 4690-4700, 2012.

[12] K. Lock, J. Zhang, J. Lu, S. H. Lee, and P. R. Crocker, “Expression of CD33-related siglecs on human mononuclear phagocytes, monocyte- derived dendritic cells and plasmacytoid dendritic cells," Immunobiology, vol. 209, no. 1-2, pp. 199-207, 2004.

[13] S. Mizrahi, B. F. Gibbs, L. Karra, M. Ben-Zimra, and F. LeviSchaffer, "Siglec-7 is an inhibitory receptor on human mast cells and basophils," The Journal of Allergy and Clinical Immunology, vol. 134, no. 1, pp. 230-233, 2014.

[14] J. Q. Zhang, G. Nicoll, C. Jones, and P. R. Crocker, "Siglec-9, a novel sialic acid binding member of the immunoglobulin superfamily expressed broadly on human blood leukocytes," The Journal of Biological Chemistry, vol. 275, no. 29, pp. 22121-22126, 2000.

[15] H. Yu, A. Gonzalez-Gil, Y. Wei et al., "Siglec-8 and Siglec-9 binding specificities and endogenous airway ligand distributions and properties," Glycobiology, vol. 27, no. 7, pp. 657$668,2017$.

[16] R. L. Schnaar, "Glycobiology simplified: diverse roles of glycan recognition in inflammation," Journal of Leukocyte Biology, vol. 99, no. 6, pp. 825-838, 2016.

[17] R. P. Schleimer, R. L. Schnaar, and B. S. Bochner, "Regulation of airway inflammation by Siglec- 8 and Siglec- 9 sialoglycan ligand expression," Current Opinion in Allergy and Clinical Immunology, vol. 16, no. 1, pp. 24-30, 2016.

[18] T. Angata and A. Varki, "Cloning, characterization, and phylogenetic analysis of siglec-9, a new member of the CD33related group of siglecs. Evidence for co-evolution with sialic acid synthesis pathways," Journal of Biological Chemistry, vol. 275, no. 29, pp. 22127-22135, 2000.

[19] J. A. Belisle, S. Horibata, J. A. A. Gubbels et al., "Identification of Siglec-9 as the receptor for MUC16 on human NK cells, B cells, and monocytes," Molecular Cancer, vol. 9, no. 1, p. 118, 2010.

[20] J. Walk, G. H. A. Westerlaken, N. O. van Uden, M. E. Belderbos, L. Meyaard, and L. J. Bont, "Inhibitory receptor expression on neonatal immune cells," Clinical and Experimental Immunology, vol. 169, no. 2, pp. 164-171, 2012.

[21] K. A. Nguyen, H. Hamzeh-Cognasse, S. Palle et al., "Role of Siglec-7 in apoptosis in human platelets," PLoS One, vol. 9, no. 9, article e106239, 2014.

[22] G. Dharmadhikari, K. Stolz, M. Hauke et al., "Siglec-7 restores $\beta$-cell function and survival and reduces inflammation in pancreatic islets from patients with diabetes," Scientific Reports, vol. 7, no. 1, article 45319, 2017.

[23] G. Foussias, G. M. Yousef, and E. P. Diamandis, "Identification and molecular characterization of a novel member of the SIGLEC family (SIGLEC9)," Genomics, vol. 67, no. 2, pp. 171-178, 2000.

[24] M. Falco, R. Biassoni, C. Bottino et al., "Identification and molecular cloning of p75/AIRM1, a novel member of the sialoadhesin family that functions as an inhibitory receptor in 
human natural killer cells," The Journal of Experimental Medicine, vol. 190, no. 6, pp. 793-802, 1999.

[25] S. Varchetta, P. Lusso, K. Hudspeth et al., "Sialic acid-binding Ig-like lectin-7 interacts with HIV-1 gp120 and facilitates infection of CD4pos T cells and macrophages," Retrovirology, vol. 10, no. 1, p. 154, 2013.

[26] Z. Zeng, M. Li, M. Wang et al., "Increased expression of Siglec9 in chronic obstructive pulmonary disease," Scientific Reports, vol. 7, no. 1, p. 10116, 2017.

[27] T. Matsumoto, N. Takahashi, T. Kojima et al., "Soluble Siglec9 suppresses arthritis in a collagen-induced arthritis mouse model and inhibits M1 activation of RAW264.7 macrophages," Arthritis Research \& Therapy, vol. 18, no. 1, p. 133, 2016.

[28] M. Saito, S. Yamamoto, K. Ozaki et al., "A soluble form of Siglec-9 provides a resistance against group B Streptococcus (GBS) infection in transgenic mice," Microbial Pathogenesis, vol. 99, pp. 106-110, 2016.

[29] Y. Tomioka, M. Morimatsu, K. Nishijima et al., "A soluble form of Siglec-9 provides an antitumor benefit against mammary tumor cells expressing MUC1 in transgenic mice," Biochemical and Biophysical Research Communications, vol. 450, no. 1, pp. 532-537, 2014.

[30] T. Yamaji, M. Mitsuki, T. Teranishi, and Y. Hashimoto, "Characterization of inhibitory signaling motifs of the natural killer cell receptor Siglec-7: attenuated recruitment of phosphatases by the receptor is attributed to two amino acids in the motifs," Glycobiology, vol. 15, no. 7, pp. 667-676, 2005.

[31] A. Varki and T. Angata, "Siglecs-the major subfamily of I-type lectins," Glycobiology, vol. 16, no. 1, pp. 1R-27R, 2006.

[32] M. S. Alphey, H. Attrill, P. R. Crocker, and D. M. F. van Aalten, "High resolution crystal structures of Siglec-7. Insights into ligand specificity in the Siglec family,", Journal of Biological Chemistry, vol. 278, no. 5, pp. 3372-3377, 2003.

[33] T. Yamaji, T. Teranishi, M. S. Alphey, P. R. Crocker, and Y. Hashimoto, "A small region of the natural killer cell receptor, Siglec-7, is responsible for its preferred binding to alpha 2,8-disialyl and branched alpha 2,6-sialyl residues. A comparison with Siglec-9," Journal of Biological Chemistry, vol. 277, no. 8, pp. 6324-6332, 2002.

[34] S. A. Malaker, K. Pedram, M. J. Ferracane et al., "The mucinselective protease StcE enables molecular and functional analysis of human cancer-associated mucins," Proceedings of the National Academy of Sciences of the United States of America, vol. 116, no. 15, pp. 7278-7287, 2019.

[35] R. L. Szabady and R. A. Welch, "StcE Peptidase and the StcELike Metalloendopeptidases," in Handbook of Proteolytic Enzymes (Third Edition), N. D. Rawlings and G. Salvesen, Eds., pp. 1272-1280, Academic Press, 2013.

[36] J. Y. Shao, W. W. Yin, Q. F. Zhang et al., "Siglec-7 defines a highly functional natural killer cell subset and inhibits cellmediated activities," Scandinavian Journal of Immunology, vol. 84, no. 3, pp. 182-190, 2016.

[37] S. Varchetta, D. Mele, A. Lombardi et al., "Lack of Siglec-7 expression identifies a dysfunctional natural killer cell subset associated with liver inflammation and fibrosis in chronic HCV infection," Gut, vol. 65, no. 12, pp. 1998-2006, 2016.

[38] P. Rosenstock, R. Horstkorte, V. S. Gnanapragassam, J. Harth, and H. Kielstein, "Siglec-7 expression is reduced on a natural killer (NK) cell subset of obese humans," Immunologic Research, vol. 65, no. 5, pp. 1017-1024, 2017.
[39] A. F. Carlin, S. Uchiyama, Y. C. Chang, A. L. Lewis, V. Nizet, and A. Varki, "Molecular mimicry of host sialylated glycans allows a bacterial pathogen to engage neutrophil Siglec-9 and dampen the innate immune response," Blood, vol. 113, no. 14, pp. 3333-3336, 2009.

[40] J. E. Hudak, S. M. Canham, and C. R. Bertozzi, "Glycocalyx engineering reveals a Siglec-based mechanism for NK cell immunoevasion," Nature Chemical Biology, vol. 10, no. 1, pp. 69-75, 2014.

[41] E. O. Long, "Regulation of immune responses by inhibitory receptors," Advances in Experimental Medicine and Biology, vol. 452, pp. 19-28, 1998.

[42] T. Avril, H. Floyd, F. Lopez, E. Vivier, and P. R. Crocker, "The membrane-proximal immunoreceptor tyrosine-based inhibitory motif is critical for the inhibitory signaling mediated by Siglecs-7 and -9, CD33-related Siglecs expressed on human monocytes and NK cells," Journal of Immunology, vol. 173, no. 11, pp. 6841-6849, 2004.

[43] G. Nicoll, T. Avril, K. Lock, K. Furukawa, N. Bovin, and P. R. Crocker, "Ganglioside GD3 expression on target cells can modulate NK cell cytotoxicity via siglec-7-dependent and -independent mechanisms," European Journal of Immunology, vol. 33, no. 6, pp. 1642-1648, 2003.

[44] E. Brunetta, M. Fogli, S. Varchetta et al., "The decreased expression of Siglec-7 represents an early marker of dysfunctional natural killer-cell subsets associated with high levels of HIV-1 viremia," Blood, vol. 114, no. 18, pp. 3822-3830, 2009.

[45] E. Brunetta, K. L. Hudspeth, and D. Mavilio, "Pathologic natural killer cell subset redistribution in HIV-1 infection: new insights in pathophysiology and clinical outcomes," Journal of Leukocyte Biology, vol. 88, no. 6, pp. 1119-1130, 2010.

[46] P. R. Crocker, "Siglecs in innate immunity," Current Opinion in Pharmacology, vol. 5, no. 4, pp. 431-437, 2005.

[47] M. Z. Zulu, K. K. Naidoo, Z. Mncube et al., "Reduced expression of Siglec-7, NKG2A, and CD57 on terminally differentiated $\mathrm{CD} 56^{-} \mathrm{CD} 16^{+}$natural killer cell subset is associated with natural killer cell dysfunction in chronic HIV-1 clade C infection," AIDS Research and Human Retroviruses, vol. 33, no. 12, pp. 1205-1213, 2017.

[48] Z. Zou, A. Chastain, S. Moir et al., "Siglecs facilitate HIV-1 infection of macrophages through adhesion with viral sialic acids," PLoS One, vol. 6, no. 9, article e24559, 2011.

[49] J. Mikulak, C. Di Vito, E. Zaghi, and D. Mavilio, "Host immune responses in HIV-1 infection: the emerging pathogenic role of Siglecs and their clinical correlates," Frontiers in Immunology, vol. 8, p. 314, 2017.

[50] Y. L. Li, Q. F. Zhang, W. W. Yin, and D. Z. Zhang, "Reduced frequency of natural killer cell on siglec- $7^{+}$is associated with progression of hepatitis B virus-related cirrhosis," Zhonghua Gan Zang Bing Za Zhi, vol. 26, no. 6, pp. 420-425, 2018.

[51] D. Zhao, X. Jiang, Y. Xu et al., "Decreased Siglec-9 expression on natural killer cell subset associated with persistent $\mathrm{HBV}$ replication," Frontiers in Immunology, vol. 9, p. 1124, 2018.

[52] M. Fukuda, "Possible roles of tumor-associated carbohydrate antigens," Cancer Research, vol. 56, no. 10, pp. 2237-2244, 1996.

[53] D. Ghaderi, R. E. Taylor, V. Padler-Karavani, S. Diaz, and A. Varki, "Implications of the presence of N-glycolylneuraminic acid in recombinant therapeutic glycoproteins," Nature Biotechnology, vol. 28, no. 8, pp. 863-867, 2010. 
[54] A. Paul and V. Padler-Karavani, "Evolution of sialic acids: implications in xenotransplant biology," Xenotransplantation, vol. 25, no. 6, article e12424, 2018.

[55] V. Padler-Karavani, N. Hurtado-Ziola, M. Pu et al., "Human xeno-autoantibodies against a non-human sialic acid serve as novel serum biomarkers and immunotherapeutics in cancer," Cancer Research, vol. 71, no. 9, pp. 3352-3363, 2011.

[56] O. M. Pearce and H. Laubli, "Sialic acids in cancer biology and immunity," Glycobiology, vol. 26, no. 2, pp. 111-128, 2016.

[57] T. Pham, C. J. Gregg, F. Karp et al., "Evidence for a novel human-specific xeno-auto-antibody response against vascular endothelium," Blood, vol. 114, no. 25, pp. 5225-5235, 2009.

[58] Y. Kawasaki, A. Ito, D. A. Withers et al., "Ganglioside DSGb5, preferred ligand for Siglec-7, inhibits NK cell cytotoxicity against renal cell carcinoma cells," Glycobiology, vol. 20, no. 11, pp. 1373-1379, 2010.

[59] N. Hashimoto, S. Ito, A. Tsuchida et al., "The ceramide moiety of disialoganglioside (GD3) is essential for GD3 recognition by the sialic acid-binding lectin SIGLEC7 on the cell surface," The Journal of Biological Chemistry, vol. 294, no. 28, pp. 1083310845, 2019.

[60] Z. Ghasemi, R. Dinarvand, F. Mottaghitalab, M. EsfandyariManesh, E. Sayari, and F. Atyabi, "Aptamer decorated hyaluronan/chitosan nanoparticles for targeted delivery of 5fluorouracil to MUC1 overexpressing adenocarcinomas," Carbohydrate Polymers, vol. 121, pp. 190-198, 2015.

[61] P. Brossart, A. Schneider, P. Dill et al., "The epithelial tumor antigen MUC1 is expressed in hematological malignancies and is recognized by MUC1-specific cytotoxic T-lymphocytes," Cancer Research, vol. 61, no. 18, pp. 6846-6850, 2001.

[62] V. Apostolopoulos, L. Stojanovska, and S. E. Gargosky, "MUC1 (CD227): a multi-tasked molecule," Cellular and Molecular Life Sciences, vol. 72, no. 23, pp. 4475-4500, 2015.

[63] S. Tanida, K. Akita, A. Ishida et al., "Binding of the sialic acidbinding lectin, Siglec-9, to the membrane mucin, MUC1, induces recruitment of $\beta$-catenin and subsequent cell growth," The Journal of Biological Chemistry, vol. 288, no. 44, pp. 31842-31852, 2013.

[64] R. Beatson, V. Tajadura-Ortega, D. Achkova et al., “The mucin MUC1 modulates the tumor immunological microenvironment through engagement of the lectin Siglec-9," Nature Immunology, vol. 17, no. 11, pp. 1273-1281, 2016.

[65] H. Prescher, M. Frank, S. Gutgemann et al., "Design, synthesis, and biological evaluation of small, high-affinity Siglec-7 ligands: toward novel inhibitors of cancer immune evasion," Journal of Medicinal Chemistry, vol. 60, no. 3, pp. 941-956, 2017.

[66] S. Varchetta, E. Brunetta, A. Roberto et al., "Engagement of Siglec-7 receptor induces a pro-inflammatory response selectively in monocytes," PLoS One, vol. 7, no. 9, article e45821, 2012. 